aus allen einfach und doppelt brechenden Stoffen. Bei ersteren sind dieselben aus oben genannten Gründen im Polarisationsapparate nicht sichtbar, bei den doppelt brechenden erscheinen sie aber, weil beide Strahlen diese Farben zeigen, der abgelenkte wie der den Analysator trefiende. Beim Umdrehen des Analysators oder Polarisators oder des Objectes selber geht die Farbe in eine andere über, erreicht jene $180^{\circ}$, allemal in die Complementarfarbe: Blättchen von gleichmässiger Dicke zeigen gleichzeitig nur eine Farbe, jede Abweichung von jener bewirkt eine andere Färbung. Prachtvolle Bilder in allen prismatischen Farben erhält man beim Krystallisiren von doppelt brechenden Stoffen durch Verdunsten aus Lösungen auf Objectträgern (Kaliumchlorat, Salicin, Hippursäure) und beim Betrachten vieler organischer Gebilde von zarter Structur. Durch in bestimmten Richtungen zu ihren Axen geschliffene Krystallblättchen erhält man Farben in gewissen kreisförmigen oder elliptischen Figuren, welche sich bei einaxigen Krystallen wesentlich anders verhalten als bei zweiaxigen. An den Stellen, welche den Elasticitätsverhältnissen entsprechend keine interferirende Strahlen durchlassen, sind die farbigen Bilder von Zonen in Gestalt von symmetrischen Kreuzen oder Curven durchbrochen. Bei parallelen Nicol'schen Prismen erscheinen dieselben durchsichtig farblos, bei gekreuzten schwarz. Viele organische Gebilde zeigen diese Zonen gleichfalls in charakteristischer Form, z. B. die Stärkemehlarten. Das Nähere hierüber muss dem Fachstudium überlassen bleiben.

(Fortsetzung folgt.)

\title{
Zur Gechichte des Chinidins und Cinchonidins und Normirung der Nomenclatur dieser Chinaalkaloïde durch den chinologischen Congress in Amsterdam (13. April 1877).
}

Von Dr. G. Kerner.

Es giebt wenig Körper in der organischen Chemie, welche seit ihrer Entdeckung so oft unabsichtlich und absichtlich ron $\mathrm{Na}$ mensveränderungen heimgesucht worden sind, als die dem Chinin isomere Base "Chinidin" und das dem Cinchonin isomere "Cincho- 
nidin". Die Ursache hiervon liegt theils in dem Umstande, dass Forscher verschiedener Nationalitäten zu verschiedenen Zeiten diese Basen immer wieder aufs Neue entdeckten und bona fide mit andern Namen belegten, ja das Cinchonidin „Chinidin “ nannten, theils ganz besonders in dem lange Jahre hindurch (hauptsächlich von 1854-1874) bestandenen Usus des Handels unter der Bezeichnung "Chinidin. sulfuric." in verschiedenen Verhältnissen zusammenkrystallisirtes oder zusammengemischtes Chinaalkaloïdsulfat zum Verkaufe zu bringen. Auf diese Weise gelangten selbst in die Universitätssammlungen und Industrie-Ausstellungen Präparate, welche nicht die Salze unvermischter Chinabasen darstellten und man beging mehrfach den Fehler, solche nicht reine Chinidine als besondere Chinidinmodificationen anzusehen.

Ich habe selbst in Folge dessen (1862) in meiner Charakteristik der Chinaalkaloüde irrthümlich 2 Modificationen der Base, als $\alpha$ nnd $\gamma$ Chinidin ${ }^{1}$ aufgeführt, von welchen Erstere wohl nur ein mit Cinchonidin verunreinigtes, die Letztere ein chininhaltiges Chinidin war; das wahre Chinidin nannte ich damals „, $\beta$ Chinidin“, charakterisirte es genügend und führte in die deutsche Nomenclatur diese Bezeichnung dafür ein. ${ }^{2}$ Koch nannte die Base

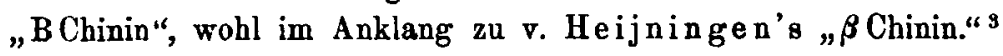

Während man nun in England und Frankreich bereits begann, das in Deutschland " $\beta$ Chinidin" und BChinin" genannte wahre Chinidin mit seinem historisch richtigen Namen, d. h. einfach mit "Chinidin" zu bezeichnen, kam schliesslich in letzter Linie Hesse 4 im Jahre 1865 mit dem Vorschlage, das Alkaloï „Conchinin“ zu nennen. Seine Absicht, gegenüber der damals, namentlich in Deutschland, missbräuchlichen Anwendung der Bezeichnung "Chinidin. sulfuric." (für Sulfate gemischter Nobenalkaloïde der Chinarinde) ein genau charakterisirtes Alkaloïd hiermit dem Begriffe

1) Leider besass ich später keine Proben der bezüglichen Präparate mehr, um auf Grund wiederholter Untersuchungen die erstmaligen irrthümlichen Bezeichnungon zu annulliren.

2) Wären mir damals die Originalabhandlungen von Pasteur, v. Heijningen und de Vrij bekannt gewesen, hätte ich zu jener Zeit die Frage schon ge-

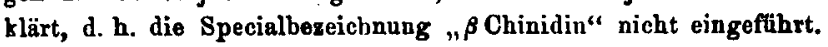

3) Ann. d. Ch. u. Ph. (1849). Bd. 72. p. 303.

4) 1865. Ann. d. Ch. u. Ph. Bd. 135, S. 325; 1868. Ibidem Bd. 146, S. 357. 
nach neu zu pröcisiren, mag eine recht gute gewesen sein, war aber eben so unpraktisch, als hindernd für die definitive Klärung der Frage, namentlich weil er gleichzeitig das Cinchonidin (die Isomerie des Cinchonins) wieder Chinidin nannte und erst einige Zeit später den ihm geradezu schädlich erscheinenden Namen Chinidin ganz abwarf, statt den einzig richtigen Griff zu thun, die missbräuchliche Benutzung des Namens zu bekämpfen und für das einzige, dem Chinin isomere, genuine Alkaloïd (krystallisirbare Rindeneduct), den gewiss sprachlich bezeichnendsten, nach historischem Rechte (d. h. vom ersten Entdecker gegebenen) nnd der Pasteur'schen Charakteristik passendsten Namen "Chinidin“ ebenfalls anzunehmen. Wenn dies noch nicht in den ersten Jahren (1865-1872) geschah, ist es allerdings damit zu rechtfertigen, dass zu jener Zeit in Deutschland immer nooh die Bezeichnung

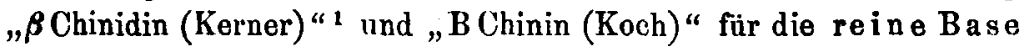
(und ihre Salze) mitgeführt wurden; später aber konnte nach dem Vorgange französischer, englischer und holländischer Chemiker und anch nach der Bezeichnung der Handelswaare in England, Frankreich, Amerika etc. keinerlei $\mathrm{Zw}$ eifel mehr darüber bestehen, welches Alkaloud (in reinem Zustande) man mit dem Namen "Chinidin" belegt haben, beziehungsweise belegt lassen wollte. In Pharmacopöen und officiellen Rapporten (so von B. Moens in den Analysen-Tabellen der holländischen Regierungsrinden) wird die fragliche reine Base seit geraumer Zeit ebens o benannt.

1) Im Jahre 1869 (Pfüger's Archiv d. Physiologic Bd. II, S. 233) habe ich mich allerdings in Anerkennung Hesse's sonst fleissiger $\Delta$ rbeiten und hauptsächlich am meinestheils nicht Prioritätsechte balber dic Vereinfachung der Nomenclatur aufzuhalten, damit einverstanden erklärt das Alkaloïd "Conchinin“ zu nennen; es zeigte sich aber bald, dass man im Handel für die reine Base in Deutschland die Bezeichnung $\beta$ Chinidin nicht abstossen wollte, so dass ich Letztere immer als Synonym gleichzeitig führte und schliesslich das "Conchinin" wieder aufgab. Noch Schrage (Archiv der Pharmacie 1878, Bd. 210, S. 25) nennt es in Achtung meiner Prioritätsrechte gegenüber dem Conchinin, wioder $\beta$ Chinidin, wodurch er sich für seine interessante Arbeit Hesse's Tadel und die grundfalsche Belehrung zuzog, dass er dann auch nach Kerner das Cinchonidia „, Chinidin “ nennen müsste. (Ibid. p. 485). -

Nicht unerwähnt möchte ich bei dieser Gelegenheit lassen, dass sich in meinor angezogenen Abhandlung (Pfl. Archiv) p. 233, Note 3 ein Druckfehler (resp. Schreibfebler) eingeschlichen hat. Es soll dort natürlich Conohinin ( $\beta$ Chinidin) statt Cinchonidin heissen. 
Hesse fing aber nun erst recht an sich auf den Namen „Conchinin" zu versteifen und man begegnet nun in seinen Veröffentlichungen allerlei Anstrengungen, um lediglich zu Gunsten seines verdrehten Conchinins („durch Versetzung der Vocale im Worte Cinchonin ") in gesuchtester Weise die Berechtigung allgemeiner Gültigkeit der Pasteur'schen Nomenclatur zu bemängeln und zu bestreiten. - Derlei Bemühungen führen natürlich auf Abwege. So behauptete z. B. Hesse schon mehrmals (zuletzt Arch. d. Ph. Bd. 209, S. 319) das Cinchonidin werde von mir " $\alpha$ Chinidin" genannt, was niemals der Fall war, da ich bekanntlich schon im Jahre 1862 unter Cinchonidin dasjenige Alkaloïd (in zwei Modificationen) auffasste, welches wasserfrei krystallisirt, daher nicht verwittert, dem Cinchonin isomer ist, mit Chlorwasser und Ammon nicht grün wird und nicht fluorescirt. ${ }^{1}$ Ich muss mich daher endlich einmal gegen diese, lediglich Hesse's Einbildung entsprosste Unterstellung verwahren. - Kurz, die nach und nach sehr klar gewordene Nomenclaturfrage wurde immer künstlich getrübt und im Fluss gehalten; gewiss nicht zum Nutzen der internationalen Wissenschaft und des Handels mit Arzneistoffen.

Es war daher sehr zeitgemäss, dass sich der im April 1877 in Amsterdam zusammengetretene internationale Chinologen-Congress unter Anderem, auf Antrag Dr. de Vrij's, auch damit beschäftigte, sich über die Nomenclatur des (hinidins zu einigen, damit ferner Niemand mehr (auch in Deutschland) über den Begriff der so benannten und ferner za benennenden Base im Zweifel sein könne. Andere Beweggründe, als die Prüfung des historischen (Prioritäts-) Rechtes des richtigen Namens, des Rechtes der Logik im Ausdruck, und schliesslich aus den beiden ersteren hervorgegangenen Rechtes der internationalen Gewohnheit per majora konnten wahrlich nicht maassgebend sein. Es war ausser mir Niemand zugegen, der unter dem Namen Chinidin etwas anderes, als die fragliche, einzige

1) Ioh habe fur genaue Beobachtung ron Fluorescenz-Erscheinungen ein Instrument construirt, das ich "Fluorescop" nannte. Hesse belehrt nun neuerdings darüber, dass der Nachweis von Chinin mit dem „Fluorescop" unter Umständen nicht zulässig sein könne, wegen Anwesenheit von Chlormetallen etc. Dase ich Alles dies, nnter Ertheilung genaner Vorschrift zur Vermeidung eines solchen Irrthums, bereits 1869 (Pflüger's Archiv, Bd. II, p. 235-238) ausfithrlich bei Beschreibung des "Fluorescop" exörtert, hat H. nat ïrlich rein zu fällig nicht erwähnt. 
Chininisomerie verstand (wenigstens wurde nichts Gegentheiliges geänssert), auch ich war längst gerne bereit, den Beinamen „ $\beta$ Chinidin" definitiv abzulegen und hatte darum keinerlei Veranlassung, für den jüngst- und nachgeborenen Namen "Conchinin“, zum Besten von Hesse's Nomenclatur-Liebhaberei, mehr als zweifelhafte Rechte geltend zu machen.

Beschlossen wurde daher, dass man ferner unter dem Namen "Chinidin", nach Pasteur, ausschliesslioh die dem Chinin isomere, krystallisirbare und verwitternde, das charakteristische, schwerlösliche Jodhydrat bildende Chinabase begreifen und bezeichnen wolle.

Die Angelegenheit konnte hiermit füglich ihr endliches Ende gefunden haben und auch Hesse sich der Entscheidung des Congresses anbequemen, aber mit Nichten. Er schliesst seinen letzten Artikel über Chinaalkaloïde mit folgender Philippica:

„Der Fall des Missverstehens ist aber sofort möglich, wenn wir uns an den bezüglichen Beschluss des chinologischen Congresses (Amsterdam am 13. Apr. 1877) halten wollen, nach welchen die Nomenclatur Pasteurs empfohlen wird, da nemlich unter Chinidin eine ganze Reihe von Chinaalkaloiden verstanden wird und zudem die Definition, welche Pasteur von seinem Cinchonidin gegeben hat, sich auf zwei Alkaloide erstreckt. Dies hätte man in dem betreffenden Congresse wohl auseinander setzen dürfen, ehe man nach zwei dürftigen Bemerkungen den bezüglichen Antrag annahm. - Uebrigens möchte ich mir erlauben anzudeuten, dass durch Congressbeschlüsse wohl Vorurtheile und dergleiehen sanctionirt werden können, was von der Qualification der Mitglieder des betreffenden Congresses abhängt, dass hingegen durch derartige Abmachungen der exacten wissenschaftlichen Forschung keine Schranken gesetzt werden können. ${ }^{1 “}$

„Was endlich noch die Behauptung betrifft, welche Schaer in dem genannten Congresse vorbrachte, dass nemlich die meisten Pharmacologen sich in fraglichen Fällen der Nomen-

1) Es ist in der That mehr als richtig, dass durch das „Vorurtheil “ der Nichtannahme des unberechtigten Namens Conchinin seitens des Congresses eine exacte wisscnschaftliche Forschung mehr erleichtert, als besclräukt wird. 
clatur Pasteurs bedienen, so kann ich' leider diese Behauptung nur bestätigen (sic!), allein ich habe andererseits bis lang die Hoffnung gehabt und habe sie noch, dass sich diese Männer dem Fortschritte, ${ }^{1}$ welcher auf dem chemischen Gebiet der Chinologie gemacht wird, auf die Dauer wohl nicht entziehen können."

Dass Hesse, aus Mangel an stichhaltigen Gründen, nun versucht, die Qualification einer Anzahl ihm in Wissenschaft und Specialerfahrung mindestens gleichwerthiger Männer zu verdächtigen, erhöht sicher seine Verdienste nicht, beraubt aber gerade ihn, jedem Unbetheiligten gegenuiber, der Qualification nüchterner $0 b$ jectivität in der Beurtheilung dieser einfachen Frage, welche von der andern Seite durchaus sachlich behandelt und entschieden worden ist. Wiederum begegnen wir zwei Angaben zur Vertheidigung seines alleinstehenden Beharrens auf dem Namen Conchinin, welche durchaus unrichtig sind. Die Möglichkeit des Missverständnisses sei durch die Sanctionirung der Pasteur'schen Nomenclatur befestigt worden, weil 1) unter „Chinidin“ eine ganze Reihe von Chinaalkaloïden verstanden werde, und 2) die Definition, welche Pasteur von seinem Cinchonidin gegeben habe, sich auf 2 Alkaloïde beziehe.

Der erste Einwand wird schon durch die einfache Logik beseitigt, dass ja eben der Beschluss, das fragliche, genau charakterisirte, einzige dem Chinin isomere Alkaloïd definitiv und ansschliesslich „Chinidin“ zu nennen, fernere Missverständnisse unmöglich machen soll und unmöglich macht. Auch wenn man etwa noch dann und wann im Handel unter "Chinidin. sulfuricum" (mit oder ohne Anhängung der Bezeichnung "venale") gemischten Chinaalkaloïdsulfaten begegnen sollte, was von Jahr zu Jahr weniger der Fall ist, wirkt doch der Congressbeschluss darauf hin, auch diese Ungehörigkeit vollends zu beseitigen. Der zweite Einwurf, dass unter Pasteurs Cinchonidin 2 verschiedene Alkaloide zu begreifen seien, ist noch unbegreiflicher; denn erstens stimmt die Charakteristik, welche Hesse für das Cinchonidin giebt, zur betreffenden Charakteristik Pasteurs und zum Andern ist H.'s Einfall, das in 2 Modificationen vorkom-

1) Also Hesse's Ritte auf dem Namen Conchinin; denn um Anderes handelt es sich hier nieht. 
mende Cinchonidin ${ }^{1}$ in zwei besondere, in sich elementar verschiedene, selbständige Alkaloïde (Cinchonidin und "Homocinchonidin") zu spalten, neueren Datums als der Congress, welcher also diese Idee $\mathrm{anmöglich} \mathrm{berücksichtigen} \mathrm{konnte,} \mathrm{abgesehen}$ davon, dass dieselbe bis heute keineswegs ron anderen Seiten bestätigt, wohl aber bezweifelt wird.

Derartigen Umgehungen des wahren Sachverhaltes und der alleinstehenden Opposition gegen die beschlossene, klare Definition des Begriffes "Chinidin“ dürfte wohl am Besten begegnet werden, indem ich nachstehend einen kurzen Abriss der älteren Geschichte dieses Alkaloïds gebe. Jedermann kann dann leicht selbst finden, dass die historischen Gründe, welche den Congress

1) Ich habe bereits 1862 darauf aufmerksam gemacht, dass das Cinchonidin in mehr als einer Modification auftritt, was sich besonders aus der Kryatallisationsart der Sulfate orgiebt, ron welchen das Eine, welches ich seither' $\alpha$ Cinch onidin nenne, käsig gallertartig krystallisirt (Koch brachte dieses Cinchonidin zuerst in magnesiaartigen Stücken in den Handel), das Andere $\beta$ Cin chonidin mehr dem Chininsulfat ähnlich erscheint. Bezüglich des Vorkommens habe ich nur in so weit einige Regelmässigkeit beobachtet, dass bei der Fabrikation sich die Modification $\alpha$ mehr in den Cinchoninmutterlaugen, die Modification $\beta$ mehr als Begleiter des Chinins und im Chinoïdin findet. Concentrirte noch unreine (gefärbte) Sulfatlösungen der Modification a gebrauchen oft Wochen lang zur Krystallisation, während das Sulfat der Modification $\beta$ meist schon beim Erkalten der Lösungen anschliesst; an den reinen, getrockneten Sulfaten beider Modificationen beobachtet man aber kaum Löslichkeitsunterschiede in kaltem Wasser. Stellt man aus beiden Sulfatformen die reinen Alka lo ï de dar, so gleichen sich dieselben vollkommen in Bezug auf Kryotallisation und Löslichkeit in den gewöhnlichen Lösungsmitteln (Wasser, Alkohol, Aether, Chloroform, Ammonliquor etc.), auch konnte ich bis jetzt keine anderen Verbindungen (a usser den Sulfaten) herstellen, welche deutliche Unterschiede (in Form und Löslichkeit) zwischen beiden Modificationen erkennen lassen, besonders gleichen sie sich auch in der Schwerlöslichkeit der Monotartrate. Im Jahre 1862 glaubte ich einige Differenz in der Krystallform der Monochlorhydrate gefunden zu haben, ersah aber später, dass diese Formunterschiede zufällig sind und bei beiden Modificationen (Rhomboïdal-Octaëder und - Prismen) resultiren können.

Elementaranalytisch konnte ich bis jetzt keine Zahlen finden, die für die eine oder die andere Modification zu einer von $\mathrm{C}^{20} \mathrm{H}^{24} \mathrm{~N}^{2} \mathrm{O}$ abweichenden Formel führten und bin daher vorläufig nicht in der Lage, Hesse's neue Auffassung zu bestätigen. Würde sich Letztere aber auch noch als richtig erweisen und nicht blos als 2 Cinchonidin-Modificationen, sondern als 2 getrennte Alkaloïde su begreifen sein, so ist dies eine Frage, die jedenfalls mit der Chinidin-Nomencl at ur nichts zu than hat. 
in erster Linie bestimmten, nichts weniger als „dü rftig“ sind, wie sie Hesse zu bezeichnen beliebt.

Das Chinidin wurde im Jahre 1833 von Henry und Delondre entdeckt. Weil diese Forscher aber fanden, dass das von ihnen gefundene Alkaloïd mit Chinin isomer sei, so glaubten sie es nicht als besondere Base betrachten zu dürfen („,comme n'étant autre chose, que de l'hydrate de quinine ") und wiederriefen irrthümlich ibre Entdeckung im Jahre 1834. Hierdurch war der Name Chinidin vorübergehend vacant geworden und es war also Winkler nicht iibel zu nehmen, wenn er das von ihm im Jahre 1848 neu entdeckte Alkaloïd (jetzt Cinchonidin) mit dem Namen Chinidin belegte und damit die unabsichtliche erste Veranlassung zu der späteren Nomenclaturverwirrung gab, da nun in Folge dessen auch Leers (Annal. d. Ch. u. Ph. Bd. 82) unter diesem Namen das Cinchonidin untersuchte und beschrieb. Es kann aber keinem Zweifel unterliegen; dass Henry und Delondre wirklich ein Alkaloïd entdeckt haben, das leicht krystallisirt und dessen Krystalle verwittern. In ihrer Abhandlung vom Jahre 1834 sagen sie: „Sa crystallisation etait le resultat d'une combinaison d'eau avec elle, car en prenant 100 parties de cristaux bien privés d'humidité exterieure par une expression convenable entre le papier joseph, puis les exposant dans un bain d'huile à $130^{\circ}$, jusqu'à ce que le poids füt invariable, on a perdu pour cent 5,8 , ce qui conduit à trouver qu'il y avoit pour un atom de quinine, un atom d'ean etc." Hieraus folgt, dass $H$. und $D$. wirklich ein verwitterndes Alkaloid in Händen hatten, also nicht blos Cinchonidin (wie Hesse jetzt behauptet), da dieses wasserfrei ist. Da aber das reine krystallisirte $\mathrm{Chinidin}$ (nach der neuesten Formel: $\mathrm{C}^{20} \mathrm{H}^{24} \mathrm{~N}^{2} \mathrm{O}^{2}$ $\left.+2 \frac{1}{2} \mathrm{H}^{2} \mathrm{O}\right) 12,2 \%$ Krystallwasser enthält, so ergiebt sich, dass das neue Alkaloïd noch nicht rein war. Es war ein Gemenge ron Chinidin und Cinchonidin, was sich überdiess noch heute durch de Vrij leicht beweisen lässt, da sich derselbe im Besitze einer Probe der Originalsubstanz befindet, die er im Jahre 1855 von Delondre selbst erbalten hat.

Auch die Bemerkung von H. und D., dass das rohe Chinoïdin der Fundort für das neue Alkaloïd sei, ${ }^{3}$ stimmt mit der thatsäch-

1) Journal de Pharm. T. 19. p. 623 (1833): "Nous pensons aussi que d'après cette cironstance, elle doit se rencontrir dans les eaux mères incriatal- 
lichen Grundlage, und haben also die Entdecker schon ausgesprochen, was v. Heijningen später beschrieben hat, als er aus dem Chinoïdin des Handels Chinidin in Menge abschied und ihm (ohne Kenntniss der Arbeit von H. and D.) den Namen „ק Chinin“ beilegte. Ferner sprachen $H$. and D. ${ }^{1}$ von einem in Wasser und Alkohol löslichen Doppelsalze, welches sie durch Sättigang des Alkaloïds mit Weinstein erhieiten. Kocht man nemlich eine Mischung aus 188 Theilen Cremor Tartari und 324 Theilen Chinidin (über $100^{\circ}$ getrocknetem) mit der etwa 5 fachen Menge Wasser, so resultirt rasch und leicht eine vollkommen klare, neutrale oder schwach basische Lösung, aus der erst boim Erkalten leichtlösliches Chinidinmonotartrat (allerdings kein Kalidoppelsalz, wie H. und D. meinten) ${ }^{2}$ auskrystallisirt. Diese Eigenschaft charakterisirt in der That das $\mathrm{Ch}$ inidin, da 308 Theile Cinchonidin (beider Modificationen) in gleicher Weise mit 188 Theilen Weinstein und selbst viel mehr Wasser anhal tend gekocht, nach dem Erkalten keine Lösung geben, aus der freies Ammon irgend welche Spur von Alkaloïd fällt.

Immerhin würde es, wenn nach der Arbeit von $H$. und $D$. weiter Nichts über das von ihnen entdeckte Chinidin gesehrieben worden wäre, nicht der Mühe werth sein, über das Prioritätsrecht des Namens zu streiten, da sich die Entdeckung auf damals (1833) zwei neue Basen (ein Gemenge) bezog. Nun aber erschien im Jahre 1853 die wirklich classische Arbeit von Pasteur, wovon leider nur ein Auszug existirt, da der Autor die beabsiohtigte ausführliche Abhandlung nicht publicirt hat. Die betreffende Veröffentlichnng findet sich in den Comptes rendus etc. Tome XXXVII, séance du 25. Juillet 1853.

Der Ausgangspunkt von Pasteurs Untersuchungen war ein Gemisch von verwitternden und nicht verwitternden Krystallen, wie es damals im Handel unter dem Namen "Chinidin" vorkam. Vom ächten Chinidin sagt er:

lissbles de la préparation du sulfate de quinine et y exister dans la quino ïdin de Mr. Sertürner etc.

1) Journal do Pharmacie. T. 20. p. 157 (1834).

2) Wahrscheinlich versäumten $H$. und D. das erhaltene Salz genügend von anhängender Mutterlauge zu befreien und erhielten darum koblonsaures Kali in der Asche, woraus sie schlossen, dass es ein Doppelsalz sei. 
"Ainsi la quinidine, découverte en 1833 pr. M. M. Henry et Delondre, est toute autre chose, que ce qu'on appelle aujonrd'hui de ce nom en Allemagne et en France, et le produit allemand est très souvent mélangé en forte proportion de celui, qui a été decouvert par M. M. H. et D. . . On trouvera dans mon mémoire (leider nicht publicirt) tous les détails nécessaires sur les propriétés et la composition des deux quinidines. J'ajouterai seulement, afin de les caractériser tout de suite, quel'une d'elles, à laqizelle je conserve le nom de quinidine est hydratée, efflorescente, isomère de la quinine, dévie à droite le plan de polarisation, et possède à l'égal de son isomèrelaquinine, le caractère de la coloration verte par addition succesive de chlore et de l'ammoniaque."

Diese Charakterisirung des Chinidins ist gewiss so deutlich, dass kaum eine Verwechslung mit einem andern Chinaalkaloïd denkbar ist, um so weniger, als Pasteur die Molecularrotation ziemlich genau zu $+250^{\circ}, 75$ für gelbes Licht angegeben hat; Oudemans fand dieselbe in Jahre 1875 mit sehr vervollkommnetem Apparate und Benützung von Natriumlicht $=+255^{\circ}, 40$. Eine solche Uebereinstimmung ist natürlich ein schlagender Beweis dafür, dass der Begriff "Chinidin“ von Pasteur genau präcisirt wurde; fuir Hesse, der nun einmal gerne das Gegentheil finden möchte, war dies aber sehr genant und versuchte er die optische Bestimmung Pasteurs durch die ungegründete Behauptung zu beanstanden, die Resultate des Letztern beziehen sich auf rothes Licht, in welchem Falle die Molecularrotation auf $327^{\circ}$ steigen würde, was unmöglich ist und woraus folgen sollte, dass Pasteur nicht mit Chinidin in alkoholischer, sondern in saurer Lösung operirt habe. Oudemans hat jedoch durch die Zahlen Pasteurs selbst den Beweis geliefert, dass Hesse's Behauptung der Begründung entbehrt, so dass also nicht Pasteur, sondern Hesse sich geirrt hat. Die Lehrbücher von Wöhler (1853) und von Gerhardt (1856) nehmen schon die Definirung des "Chinidins “ nach Pasteur an.

Von dem nicht verwitternden Alkaloïd der Originalsubstanz sagt Pasteur:

"L'autre base, à laquelle je donne le nom de cinchonidine est anhydre, isomère de la cinchonine, exerce à gauche son pouvoir rotatoire et ne possède pas le caractère précité de la coloration verte. C'est elle qui est aujourd'hui 
la plus abundante dans les échantillons commerciaux. Il est toujours très facile, en exposant à l'air chaud une crystallisation récente de cinchonidine, de reconnaître si elle renferme la quinidine. Tous les cristaux de cette dernière base s'effleuriront immédiatement en conservant leurs formes et se détacheront en blanc mat sur les cristanx de cinchonidine demeurés limpides. En resumé donc, il y a dans les écorces de quinquina quatre alcalis principaux: la quinine et la quinidine, - la cinchonine et la cinchonidine.“

Es unterliegt also keinem Zweifel, dass (selbst abgesehen von der Entdeckung Henry und Delondre's) wenigstens schon im Jahre 1853 vier genan charakterisirte Haupt-China-Alkaloïde bekannt waren, und giebt es also gewiss keine Ursache, warum man, unter Aufrechterhaltung der d rei übrigen Namen, den Vierten, und zwar das mit dem Chinin isomere "Chinidin" in den Nichts ausdrückenden Namen „Conchinin" umwandeln soll. So lange Hesse die Bezeichnung "Chinidin" für das von Winckler entdeckte Alkaloïd nicht aufgeben wollte, mochte hierzu noch einige entschuldigende Veranlassung sein, seitdem or aber selbst für das Letztere den vón Pasteur gegebenen Namen "Cinchonidin " angenommen hat, verblieb doch anch für ihn kein triftiger Grund, die einzige genuine, dem Chinin isomere Base anders als mit dem historisch und sprachlich richtigsten Namen "Chinidin" zu bezeichnen.

Nenerdings hat Hesse allerdings Pasteur vorgeworfen, dass unter seinem Cinchonidin 2 Basen zu begreifen seien; so. lange aber H. die Unterscbeidungsmerkmale seines sogenannten „Homocinchoniding " und die Art der Trennung desselben vom Cinchonidin nicht näher angiebt, kann er andern Chemikern, wie Oudemanns, de Vrij und mir, nicht verübeln, wenn sie vorläufig diese „neue“ Base als sehr problematisch betrachten. ${ }^{1}$ (Vgl. auch die Note pag. 265.)

Die schöne Untersuchung Pasteurs war für den Holländer Dr. de Vrij die Veranlassung, sich speciell dem Studium der China-

1) Soeben bestätigt auch Skraup (Annalen 199, p. 359) in ausführlicher Darlegung ron Versuchen, deren Ergebnise ziemlich in allen Theilen mit meinen, nicht verötfentlichen diesbezüglichen Erfahrungen ü bereinstimmt, das Hesse's Auffassung eines "Homocinchonidins" als benondere Base unberechtigt ist. 
alkalö̈de zu widmen und hat derselbe ganz besonders die Anwendung des Polarisationsinstrumentes als sehr schätzenswerthes Hülfsmittel betrachtet und seit 1855 für alle $\mathrm{ihm}$ in die Hände gekommene Präparate benutzt. Von 1855-1857 arbeitete or in Rotterdam, von 1858-1863 in Bandong (Java) mit dem Biot'schen Apparat und seit seiner Rückkehr aus Java bis jetzt in seinem Privatlaboratorium im Haag mit dem Wild'schen Polaristrobometer. So suchte er auch frühzeitig nach dem Chinidin (Pasteur) und erhielt Ende 1855 die bezüglichen Präparate direct von den Fabrikanten Delondre und Howard (also von 2 verschiedenen Seiten).

Im Jahre 1856 erkannte er am (ächten) Chinidin zwei neue, auffällige Eigenschaften, dass dasselbe

1) mit Wasser gekocht und kochend heiss filtrirt, beim Erkalten in (verwitternden) Krystallen anschiesst,

2) mit Jodwasserstoffsüure ein sehr charakteristisches Salz bildet, das bei gewöhnlicher Temperatur mehr als 1200 Thle. Wasser zur Lösung bedarf und anch in Alkohol sehr schwer löslich ist, wenn auch mehr als in Wasser.

Die Resultate dieser Arbeiten publicirte er Febr. 1857 in Haaxman's Tijdschrift voor wetensohappelyke Pharmacie (in Auszug in Cannstadts Jahresbericht 1857, S. 43). Er gab damals schon genaue Anleitung dazu, die ron ihm entdeckte ${ }^{1}$ Eigenschaft des jodwasserstoffsauren Salzes als Reagens auf Chinidin anzuwenden, und es nimmt sich komisch aus, wenn dies 12 Jahre später als „Hesse'sche Conchininprobe“ neu aufgetischt wird.

In Java angekommen, beschäftigte sich de Vrij anfangs mit der Analyse von Rinden abgestorbener Chinabäume. Im Jahre 1859 stellte er das erste Chinin aus Javarinden dar und fand auch schon bereits, dass sich die Java Calisaya durch einen Gehalt an Chinidin (Pasteur) unterscheidet. (Reines Cinchonin, Chininoxalat and reines jodwasserstoffsaures Chinidin, ron ihm in jener Zeit bereitet, waren mit Javacinchonen auf der Londoner Ausstellung 1862 zu sehen und finden sich die Original-

1) Ungefähr zu gleicher Zeit fand ich diese Reaction (mitgetheilt aber erst 1862); es gehört also de Vrij jedenfalls die Priorität. Hätte ich, wie de V., dessen Publication mir enst viel später bekannt wurde, Originalpräparate von Pasteurs Chinidin zar Vergleichung besessen, wäre ich nicht dazu gekommen, 1862 da reine Chiridin als besondere Modification,$\beta$ Chinidin“ aufauführen, was auf Grund der Jodkaliumureaction geschah. 
präparate noch hente im Colonial-Museum zu Harlem). Wenn nun Hesse im Jahre $1874^{1}$ diese Eigenthümlichkeit der Javacalisaya noch einmal (d. h. sogen. "Conchinin" darin) findet und sich dabei den Ausfall erlaubt, „allein die Beweisführung de Vrij's war ungenügend, weil er damals (1869) die Eigenschaften der häuflger vorkommenden Chinaalkaloïde noch zu wen ig kannte", so ergiebt sich daraus, wie ungenirt und ungerechtfertigt er Andern, zur Erhöhung des eigenen Reliefs, Verdienste abspricht. Der Spiess von "dürftigen Bemerkungen" und "ungenauen Kenntniss " kehrt sich aber von selbst um, wenn man Hesse's Entwickelung in dieser Frage historisch folgt.

Im Jahre 1865 (Annal, der Chemieetc., B. 135) nennt er erstmals das reine Chinidin "Conchinin." Liest man nun daselbst p. 333: „ein Chinidin im Sinne Pasteurs, noch endlich Stenhouse's Chinidin, zwei unter sich verschiedene Alkalö̈de, habe ich bis jetzt im käuflichen Chinidin nicht finden können", so folgt daraus, dass de Vrij doch schon viel früher (1856) in der Frage klar sah; er hatte bewiesen, dass das ihm von Howard übergebene Chinidin identisch war mit dem von Pasteur (P. gab ihm von seiner Originalsubstanz), - und, (da Stenhouse ein gleiches Präparat von Howard zu seinen Untersuchungen verwendete und damit das charakteristische weinsaure Chinidin - Antimonoxyd bereitete ${ }^{2}$ ) in Folge dessen anch mit dem von Stenhouse. Ferner sagt Hesse 1868 (Annal. d. Ch. Bd. 146): „ich bin zu der Ueberzengung gelangt, dass van Heyningen's $\beta$ Chinin ein Gemenge von Conchinin und Chinidin" (damals nannte er das Cinchonidin noch Chinidin) "gewesen ist." Abgesehen nun davon, dass van Heijningen sein $\beta$ Chinin krystallinisch dargestellt hat und die verwitternden Krystalle sehr leicht unterschieden werden können, hat de Vrij im Mai 1855 ein von van Heyningen

1) 1874. Annal. d. Chemie u. Ph. Bd, 174, S. 338.

2) Später (1868. Bd. 146, p. 368) räumt Hesse ein, dass er das Stenhouse'sche Antimonsalz desshalb früher mit Pasteurs Chinidin nicht erhalten habe, "weil grosse Krystalle des "Conchining" sich nur äusserst langsam in Brechweinstoinsolution auflöwen." Dass er, die alte Regel ,corpora non agunt nisi fluida "vergessend, versäumt hatte, die grossen Krystalle zerrieben (in feiner Zertheilung) anzuwenden, ist ein Lapsus, der passiren kann und, wenn ohrlich widerrufen, nicht monirt zu werden braucht; allein Hesse hütte daraus wohl die Lehre ziehen dürfen, dass es räthlich ist, nicht un nöthig mit Steinen zu worfen, wenn man im Glashause sitzt. 
persönlich dargestelltes Präparat erhalten, untersucht und durch die Rotation, sowie vermittelst Jodwasserstoffsäure dessen vollkommene Identität mit Pasteur's, Howard's, Stenhouse's und später auch mit meinem $\beta$ Chinidin unzweifelhaft dargethan. Die erste genaue Kenntniss rom Chinidin ist also auf de Vrij und nicht auf die Inscenirung des "Conchining" 1 zurückzuführen.

Weitere Erörterungen wird es nicht bedürfen, um zu beweisen, dass der Chinologen - Congress vollkommen berechtigt war, für das einzige, dem Chinin isomere, krystallinische Alkaloïd den Namen "Chinidin" definitiv zu bestätigen, und dass er auch, im Interesse der wissenschaftlichen Ordnung, ein Festhalten an diesem Beschlusse erwarten darf.

Die Charakteristik der vier genuinen $\mathrm{Hauptalkalö̈de} \mathrm{der}$ Chinarinden (nach der hiernach feststehenden Nomenclatur) lässt sich durch folgendes Schema übersichtlich darstellen. (Die selteneren, und die amorphen Alkaloïde, sowie die Alkaloïdderivate sind hierbei absichtlich nicht aufgefihrt).

\begin{tabular}{|c|c|c|c|}
\hline \multirow{3}{*}{$\begin{array}{c}\text { Isomere } \\
\text { Alka loï } \\
\text { von der Formel: } \\
\mathrm{C}^{80} \mathrm{H}^{24} \mathrm{~N}^{2} \mathrm{O}^{2} \\
+\mathrm{I} \mathrm{H}^{2} 0 . \\
\end{array}$} & \multicolumn{2}{|c|}{$\begin{array}{l}\text { Drehen die Polarisationsebene nach } \\
\text { Links. Bilden in Wasser sehr schwer } \\
\text { lösliche Monotartrate. }\end{array}$} & \multirow{2}{*}{$\begin{array}{c}\text { Isomere } \\
\text { A lkaloide } \\
\text { von der Formel: } \\
\mathrm{C}^{20} \mathrm{H}^{24} \mathrm{~N}^{2} \mathrm{O} .\end{array}$} \\
\hline & \multirow{3}{*}{$\begin{array}{l}\text { Chinin. } \\
\text { In Aether leicht } \\
\text { löslich. Seine mei- } \\
\text { sten Salze sind weit } \\
\text { sch werer löslich, } \\
\text { als die entsprechen- } \\
\text { den Salze der übri- } \\
\text { gen Chinaalkaloüde. } \\
\text { Bildet einen in Al- } \\
\text { k ohol schwer -lös- } \\
\text { lichen, charakteristi- } \\
\text { schen Herapathit. }\end{array}$} & \multirow{3}{*}{$\begin{array}{l}\text { Cinehonidin. } \\
\text { In Aether ehr } \\
\text { schwer lö slich. } \\
\text { Bildet derbe, grosse } \\
\text { wasserhelle Krystalle } \\
\text { als Hydrochlorat. } \\
\text { Kommt in } 2 \text { Modi- } \\
\text { ficationen vor, die } \\
\text { sich durch die Form } \\
\text { der Sulfate unter- } \\
\text { scheiden. }\end{array}$} & \\
\hline & & & \multirow{4}{*}{$\begin{array}{l}\text { Ihre ssuren Lö- } \\
\text { sungen fluo- } \\
\text { resciren } \\
\text { nieht und ge- } \\
\text { ben mit Chlor- } \\
\text { wasser und Am- } \\
\text { mon keine } \\
\text { Grünfärbung. } \\
\text { Die reinen Al- } \\
\text { kaloïde krystal- } \\
\text { lisiren asser- } \\
\text { frei und ver - } \\
\text { wittern daher } \\
\text { nicht. }\end{array}$} \\
\hline $\begin{array}{l}\text { Ihre Lösungen } \\
\text { in Sauerstoffsäu- } \\
\text { ren fl u oresci- } \\
\text { ren blau. Die } \\
\text { Lösnngen ihrer } \\
\text { Salze geben mit } \\
\text { Chlorwasser und } \\
\text { Ammon eine }\end{array}$ & & & \\
\hline $\begin{array}{l}\text { charakterioti- } \\
\text { sche Grünfär- } \\
\text { bung. Die rei- } \\
\text { nen Alkaloïde } \\
\text { bilden kryotalli- } \\
\text { nische Hydra- } \\
\text { te, welche ver- } \\
\text { wit tern. }\end{array}$ & $\begin{array}{l}\text { Chinidin. } \\
\text { In Aether s c } \mathrm{h} \text { w e } \\
\text { löslich. Bildet ein in } \\
\text { Wasser und Alkohol } \\
\text { sehr schwer lösliches, } \\
\text { krystallinisches Hy - } \\
\text { droj od a t. }\end{array}$ & $\begin{array}{l}\text { Cinchonin. } \\
\text { InAether am schwer- } \\
\text { s ten löslich. Wird } \\
\text { aus mässig verdünn- } \\
\text { ten neutralen Lösun- } \\
\text { gen durch KJ nicht } \\
\text { gefällt. Das Hydro- } \\
\text { jod at ist in Alkohol } \\
\text { leicht löslich. }\end{array}$ & \\
\hline & & & \\
\hline
\end{tabular}

1) Es liegt mir ferne, mit vorstehenden Darlegungen Hesse's wirkliche Verdienste um die Chinologio im Geringsten schmälern zu wollen, вein intenai- 